Revue internationale P.M.E.

Économie et gestion de la petite et moyenne entreprise

Revure

internationale

PME

\title{
La gestion des ressources humaines dans les petites et moyennes entreprises au Québec
}

\section{Carmelle Benoît et Marie-Diane Rousseau}

Volume 3, numéro 1, 1990

URI : https://id.erudit.org/iderudit/1007945ar

DOI : https://doi.org/10.7202/1007945ar

Aller au sommaire du numéro

Éditeur(s)

Presses de l'Université du Québec

ISSN

0776-5436 (imprimé)

1918-9699 (numérique)

Découvrir la revue

Citer cet article

Benoît, C. \& Rousseau, M.-D. (1990). La gestion des ressources humaines dans les petites et moyennes entreprises au Québec. Revue internationale P.M.E.,

3(1), 39-55. https://doi.org/10.7202/1007945ar
Résumé de l'article

$\mathrm{Au}$ cours des vingt dernières années, les études empiriques en gestion des ressources humaines ont cherché à établir les facteurs déterminants des pratiques des entreprises. Bien que l'objet de ces études soit avant tout la grande entreprise, elles mettent en lumière certaines variables discriminantes à savoir l'environnement de l'entreprise, la stratégie d'entreprise, la culture d'entreprise et la vision du dirigeant. Cet article présente quelques résultats préliminaires d'un projet de recherche en cours auprès d'un échantillon représentatif de 400 dirigeants de petites et moyennes entreprises au Québec. L'étude a pour objectif de décrire la réalité spécifique des pratiques de gestion des ressources humaines des PME et d'identifier les principaux facteurs liés à l'environnement interne et externe de l'entreprise pouvant influencer ces pratiques. 


\title{
La gestion des ressources humaines dans les petites et moyennes entreprises au Québec
}

\author{
Carmelle BENOIT \\ Marie-Diane ROUSSEAU \\ Ministère de la Main-d'œuvre et de la Sécurité du revenu du Québec
}

\begin{abstract}
RÉSUMÉ
Au cours des vingt dernières années, les études empiriques en gestion des ressources humaines ont cherché à établir les facteurs déterminants des pratiques des entreprises. Bien que l'objet de ces études soit avant tout la grande entreprise, elles mettent en lumière certaines variables discriminantes à savoir l'environnement de l'entreprise, la stratégie d'entreprise, la culture d'entreprise et la vision du dirigeant. Cet article présente quelques résultats préliminaires d'un projet de recherche en cours auprès d'un échantillon représentatif de 400 dirigeants de petites et moyennes entreprises au Québec. L'étude a pour objectif de décrire la réalité spécifique des pratiques de gestion des ressources humaines des PME et d'identifier les principaux facteurs liés à l'environnement interne et externe de l'entreprise pouvant influencer ces pratiques.
\end{abstract}

\section{ABSTRACT}

Over the past twenty years, experimental studies in the field of human resources management have sought to establish the factors determining business practices. Although most of these studies focused on large businesses, they did bring to light several differentiating variables such as the business environment, strategy and culture, and also the vision held by management. This article introduces a current preliminary data from a current research project that will survey a representative sampling of $\mathbf{4 0 0}$ managers of small and medium-sized businesses in Quebec. The purpose of the project is to describe specific human resources management practices in small and medium-sized businesses and to identify the main factors in the internal and external environment of businesses that we capable of influencing these practices.

- Carmelle Benoît, sociologue, est auteur de plusieurs publications sur les nouvelles technologies et le travail. Elle est chargée de projet pour l'étude sur la GRH dans les PME au Québec. Marie-Diane Rousseau, sociologue, a été chargée d'études et de programmes en éducation des adultes, en santé et sécurité du travail et en formation professionnelle. Adresse : Direction de la recherche, Ministère de la Main-d'œuvre, de la Sécurité du revenu et de la Formation professionnelle du Québec, 255 est, boul. Crémazie, $1^{\text {ex }}$ étage, Montréal, Québec, H2M 1L5. Téléphone : (514) 873-7426; télécopieur: (514)873-0280. 


\section{RESUMEN}

En el transcurso de los ultimos veinte anos, los estudios empiricos en gestion de los recursos humanos trataron de establecer los factores determinantes de las practicas de las empresas. Aunque su objeto es ante todo la grand empresa, dichos estudios ponen en evidencia algunas variables discriminates, a saber el entorno de la empresa, la estrategia y el ambiente social y cultural de la misma, asi como la vision del dirigente. En este articulo se exponen algunos resultados preliminares de un proyecto de investigacion que se esta llevando a cabo en Quebec con una muestra representativa de $\mathbf{4 0 0}$ dirigentes de pequenas y medianas empresas. EI estudio tieno como objetivo describir la realidad especifica de las practicas de gestion de los recursos humanos en las PyME e identificar los principales factores ligados al medio interno y externo de la empresa capaces de influenciar estas practicas. 
Au cours des deux dernières décennies, la gestion du personnel ou des ressources humaines (GRH) a faitl'objet de nombreuses publications fondées sur des études empiriques décrivant les pratiques et les systèmes de GRH adoptés par les entreprises. Nombre d'études ont proposé aussi un cadre théorique pour l'analyse de ces pratiques permettant de mieux comprendre la place et l'importance de cette fonction dans la gestion d'une entreprise (Wils, 1989; Besseyre des Horts, 1988). Cependant, peu d'études empiriques portent sur la GRH dans la petite et moyenne entreprise (PME); celles-ci demeurent pour la plupart à caractère descriptif et ne proposent pas toujours un cadre de référence donnant un sens à ces pratiques. Bien qu'une réflexion spécifique sur la gestion de la PME soit plus largement engagée, la place occupée par les ressources humaines comme objet d'étude demeure très limitée et marquée par une absence d'analyse et d'outils appropriés à la petite et moyenne entreprise (Mahé de Boislandelle, 1988).

Les études empiriques et théoriques sur la GRH dans la grande entreprise ont permis de mettre en lumière la variété des pratiques et de relier ces réalités à un ensemble de facteurs déterminants tels l'environnement de l'entreprise, la stratégie d'entreprise, la culture de l'entreprise et la vision du dirigeant.

Nous ne disposons point, à l'heure actuelle, de modèles d'analyse éprouvés pour la gestion des ressources humaines dans les PME. Les études consultées sur ce sujet révèlent que la vision du dirigeant, ses valeurs et ses objectifs jouent un rôle prédominant dans la conception et la réalisation des activités de GRH (Mahé de Boislandelle, 1988). Il convient donc, tout en s'inspirant des théories développées pour la compréhension des politiques et pratiques de GRH dans la grande entreprise, de parfaire nos connaissances de la PME en cette matière et de contribuer à l'émergence d'un cadre d'analyse qui recouvre bien leur spécificité.

Cet article a pour but de présenter un projet de recherche en cours sur la GRH dans les petites et moyennes entreprises au Québec. L'objectif de cette étude est double. D'abord, nous voulons tracer un portrait des activités de GRH dans les PME. Ensuite, nous cherchons à identifier les facteurs pouvant influencer et expliquer ces activités. Précisons aussi que cette étude, conduite par le Ministère de la Main-d'œuvre, de la Sécurité du revenu et de la Formation professionnelle du Québec, a pour fin de mieux connaître la réalité de la gestion des ressources humaines dans les PME, les problèmes et les besoins de la direction des entreprises dans ce domaine, ceci afin de mieux articuler l'intervention gouvernementale auprès de ces entreprises.

\section{Contexte de l'étude}

Des études récentes signalent l'importance croissante des PME dans l'économie du Québec. En 1984, l'ensemble des entreprises du secteur privé au Québec 
se répartissait comme suit: $91,7 \%$ de très petites entreprises (1-19 employés), $6,1 \%$ de petites entreprises (20-99 employés), 1,5\% de moyennes entreprises (100-499) et $0,6 \%$ de grandes entreprises (500 et plus). Quant au volume et à la répartition de l'emploi, il correspondait à $21,6 \%$ du total pour la très petite entreprise, à $18 \%$ pour la petite entreprise, à $17,2 \%$ pour la moyenne et à $43,1 \%$ pour la grande entreprise. On observe également, entre 1978 et 1984, la contribution prépondérante des très petites entreprises dans la création d'emploi alors que la moyenne et la grande entreprise y affichent un recul important (Laroche, 1988).

Parler de volume d'emploi et de création d'emploi nous amène rapidement à la question de la gestion des emplois et à la gestion des ressources humaines dans les PME. En effet, la contribution des PME à la création d'emploi s'accompagne aussi d'une certaine précarité des emplois, de conditions de travail inférieuresà celles de la grande entreprise, d'une main-d'œuvre plus jeune $(53,7 \%$ des jeunes travailleurs âgés entre 15 et 24 ans étaient à l'emploi d'entreprises de moins de 100 employés, en 1984) (Laroche, 1988).

Les spécialistes de la PME au Québec consultés dans le cadre de cette étude et dont les informations ont été consignées dans un rapport préliminaire (Benoit et Rousseau, 1989) s'accordent généralement pour dire que celle-ci gère le personnel de façon très différente de la grande entreprise. Elle accorderait moins d'importance à la GRH, les activités dominantes étant de gérer les contrats et d'organiser la production de biens ou de services.

Étant peu outillée pour faire de la planification, n'ayant pas toujours les moyens et le temps voulu pour exercer ses capacités d'anticipation, la PME serait par le fait même plutôt en position de réaction que de prévision. Il en serait ainsi pour la planification des besoins de main-d'œuvre (embauche, promotion, mutation et départ) dont la portée serait plutôt restreinte et de nature informelle.

L'un des défis administratifs des dirigeants de PME le plus souvent signalé est celui du recrutement d'une main-d'œuvre qualifiée (Lauzier, 1987; Gasse, 1982), ce qui s'associe parfois à des problèmes importants de rétention et de roulement de la main-d'œuvre. Certains auteurs suggèrent que cette difficulté de trouver et de maintenir les travailleurs qualifiés serait reliée aux conditions de travail offertes, généralement moins avantageuses que celles de la grande entreprise. Nous connaissons très peu la dynamique de la détermination et de la révision des salaires dans les PME au Québec. Entre 1978 et 1984, les conditions salariales des employés des PME se sont grandement détériorées passant de $81,2 \%$ à $74,5 \%$ du salaire des grandes entreprises dans le cas des très petites entreprises ( 1 - 19 employés) et de $84,3 \%$ à $79,8 \%$ pour la petite entreprise ( $20-99$ employés). Les pratiques de rémunération dans la PME méritent donc d'être mieux connues.

Certaines études sur la formation (Tremblay, 1990; Thompson, 1989) tendent à démontrer la faible importance des activités de formation organisées dans les entreprises au Québec. Cependant, aucune étude ne permet d'établir les politiques 
et pratiques de formation des dirigeants de PME. Quelles sont les perceptions de ces dirigeants à l'égard de la formation, quels sont les obstacles rencontrés dans la réalisation de ces activités et quels sont leurs besoins?

Par ailleurs, les spécialistes des relations de travail et du développement organisationnel indiquent qu'outre et avant même les pratiques purement administratives de la GRH (planification des effectifs, recrutement, rémunération, formation,...), ce sont les pratiques en matière de climat organisationnel du travail qui mettent le plus en péril la PME. Ainsi, la performance de ces entreprises tiendrait à la mobilisation du personnel, à la motivation et la satisfaction au travail, à l'information et la participation des employés et à une bonne communication dans l'entreprise.

\section{Objet de l'étude}

C'est dans ce contexte général que s'inscrit le projet de recherche sur la gestion du personnel dans la PME au Québec. Nous avons choisi de faire une analyse descriptive de certaines activités de GRH et de les explorer en relation avec des caractéristiques internes et externes de l'entreprise pour mieux cerner la place et l'importance de la GRH dans les PME.

Dans une approche centrée sur les activités, la GRH se définit généralement comme l'ensemble des activités d'acquisition, de développement et de conservation des ressources humaines visant à fournir aux organisations de travail une maind'œuvre productive, stable et satisfaite (Bélanger, 1988). Par ailleurs, une vision systémique de la GRH nous présente les activités de gestion des ressources humaines dans leur caractère d'interdépendance dont les effets se conjuguent les uns avec les autres dans la réalisation de résultats déterminés, dans un environnement donné (Bélanger, 1988). Cette orientation systémique a eu comme prolongement, dans la dernière décennie, une vision stratégique des ressources humaines qui établit un lien entre les stratégies d'entreprise et les stratégies de GRH. Plus récemment encore, la thèse que proposent certains auteurs (Wils, 1989) est à l'effet que les systèmes de GRH ne varient pas systématiquement selon le type de stratégie d'entreprise. D'autres facteurs explicatifs tels les catégories d'employés et le système de valeurs des dirigeants doivent être retenus; la stratégie sociale devient alors un facteur aussi déterminant que la stratégie de développement de l'entreprise.

Les analyses empiriques et théoriques dans le champ de la gestion des ressources humaines nous fournissent des cadres de référence utiles pour la compréhension de ce phénomène. C'est à partir de ces connaissances que nous avons construit un modèle conceptuel provisoire pour l'analyse des activités de GRH dans les PME. Nous disons, provisoire, puisque, d'une part, l'analyse théorique de la GRH en PME ne fait qu'émerger et d'autre part parce que le cadre théorique qui fonde notre choix de variables et d'hypothèses sera soumis à l'observation des pratiques en GRH que nous supposons complexes, variées et mouvantes. 
Le modèle conceptuel retenu comprend, comme variable principale et dépendante, le système ou les activités de GRH et comme variables explicatives les caractéristiques liées à l'environnement interne et externe de l'entreprise.

Dans un premier temps, nous voulons décrire les principales activités de GRH (planification, recrutement, formation, motivation, information, etc.) sous l'angle des attitudes, des politiques, des pratiques, des problèmes vécus et leurs causes, ainsi que des besoins de la direction des entreprises.

Ainsi, nous cherchons à connaître :

- les objectifs et attitudes du dirigeant à l'égard de chacune des activités (importance accordée à la planification, à la formation, etc, et les objectfis poursuivis);

- les principales politiques et pratiques de GRH dans l'entreprise;

- les principaux problèmes rencontrés dans la réalisation de ces activités ainsi que les causes à ces problèmes;

- les besoins des entreprises pour accomplir ces activités (aide privée ou publique, information, formation, etc.).

Outre l'analyse descriptive des activités de GRH, la mise en relation de ces activités avec un ensemble de variables à caractère explicatif nous apparait essentielle pour comprendre la stratégie de GRH des entreprises. Il s'agit ici de caractéristiques liées à l'environnement interne et externe de l'entreprise considérées comme des facteurs d'influence et de détermination du système ou des activités de GRH.

Le tableau 1 suivant schématise l'ensemble de ces variables.

Nous ne prétendons pas pouvoir valider ou invalider l'ensemble de ce modèle. Notre étude vise à préciser la nature des liens entre le système de GRH et certains aspects de l'environnement interne de l'entreprise. Elle cherche à répondre à une question : à quoi peut-on attribuer les différences de pratiques de GRH dans les PME ? À la stratégie d'entreprise et à la philosophie de gestion ? Au profil et à la vision du dirigeant ? Aux caractéristiques du personnel ? Aux caractéristiques générales de l'entreprise?

\section{Méthodologie de l'étude}

Une série d'entretiens (33) ont d'abord été réalisés auprès de personnes ressources du milieu, soit des chercheurs ou intervenants dans le domaine de la GRH et de la PME provenant des milieux universitaire, syndical, patronal et gouvernemental, ainsi que des experts en gestion-conseil. A partir de cet état de connaissances sur la GRH dans les PME québécoises, nous avons en partie arrêté un certain nombre de choix méthodologiques. Ces entretiens nous ont permis, par exemple, de déterminer 
Tab. 1 Influence de l'environnement interne et externe de l'entreprise sur la stratégle de GRH (Modèle provisoire)

\begin{tabular}{|l|}
\hline \multicolumn{1}{|c|}{$\begin{array}{c}\text { ENVIRONNEMENT INTERNE } \\
\text { DE L'ENTREPRISE }\end{array}$} \\
\hline $\begin{array}{l}\text { Caractéristique de l'entreprise : } \\
\text { taille, secteur, région, âge de l'entre- } \\
\text { prise, âge de la gestion, innovation, } \\
\text { informatisation, performance écono- } \\
\text { mique }\end{array}$ \\
\hline $\begin{array}{l}\text { Stratégie d'entreprise et philoso- } \\
\text { phie de gestion }\end{array}$ \\
\hline $\begin{array}{l}\text { Profil du dirigeant : âge, sexe, } \\
\text { scolarité, expérience, attitudes, } \\
\text { valeurs et vision }\end{array}$ \\
\hline \\
\hline $\begin{array}{l}\text { Cactóristiques du personnel : } \\
\text { occupation, áge, sexe, ancienneté, } \\
\text { revendications et comportements }\end{array}$ \\
\hline
\end{tabular}

\begin{tabular}{|l|}
\hline \multicolumn{1}{|c|}{ STRATEGIE DE GRH } \\
\hline Objectifs économiques et soclaux \\
\hline $\begin{array}{l}\text { Moyens : activités de GRH } \\
\text { Planification : évaluation du rende- } \\
\text { ment } \\
\text { Recrutement : motivation } \\
\text { Formation : information } \\
\text { Rémunération : participation }\end{array}$ \\
\hline
\end{tabular}

\section{ENVIRONNEMENT EXTERNE DE L'ENTREPRISE}

Environnement économique :

Marché du travail, conjoncture,

compétition et concurrence

Environnement technologique :

Diffusion des nouvelles technologies,

état des innovations et de la recher-

che et développement

Environnement social : valeurs

socio-culturelles, idéologies de

gestion, dominantes (quête de la

quaitié, mobilisation des ressources

humaines)

Environnement léglslatif : lois, règlements, système d'aide gouvernementale 
avec certitude que l'outil le plus approprié pour recueillir des informations auprès des entreprises était un questionnaire administré en entrevue directe.

L'étude s'appuie donc sur une enquête, par entrevue en face-à-face, d'une durée de deux heures, au moyen d'un questionnaire de 85 questions administré auprès du dirigeant d'entreprise et des personnes responsables du personnel.

L'unité d'observation est l'entreprise, définie comme une entité juridique et financière distincte qui peut comprendre plusieurs établissements. Notre choix entre l'entreprise et l'établissement, comme base de sondage, s'appuie sur l'importance de connaître la gestion de la GRH à l'échelle de l'entreprise et non au niveau de l'une ou l'autre de ces composantes.

Pour les fins de la présente étude, nous avons retenu comme définition d'une petite et moyenne entreprise celle proposée par le ministère de l'Industrie, du Commerce et de la Technologie du Québec (1988) qui se réfere au nombre d'employés. On définit alors comme PME les entreprises du secteur manufacturier qui comptent moins de 200 employés ( 10 à 49 employés pour les petites entreprises et 50 à 199 employés pour les moyennes entreprises) et celles des autres secteurs qui comptent moins de 100 employés ( 0 à 49 employés pour les petites entreprises et 50 à 99 employés pour les moyennes entreprises).

L'échantillon prélevé est constitué de 400 petites et moyennes entreprises du secteur privé au Québec, à l'exclusion des entreprises du secteur primaire, de la construction, et des secteurs publics et parapublics.

Le plan d'échantillonnage retenu est un plan stratifié. Les secteurs d'activités des grands secteurs secondaire et tertiaire ont été regroupés selon le niveau de technicité et de scolarité des employés. Un indice synthétique de ces deux critères a été construit à partir de la proportion des cadres, des professionnels et des techniciens (indice de technicité) et de la proportion des employés ayant fait des études postsecondaires (indice de scolarité). Cette stratification correspond à l'hypothèse que certains auteurs suggèrent, à savoir que la gestion des ressources humaines tend à varier selon les catégories d'employés (Schuler et Jackon, 1987; Wils, Labelle et Le Louarn, 1988).

En rapport avec le niveau de technicité et de scolarité des secteurs d'activité, cinq strates ont donc été définies dont deux dans le secteur secondaire (niveau fort et moyen, niveau faible) et trois dans le secteur tertiaire (niveau fort, niveau moyen, niveau faible). Il est prévu de compléter 80 entrevues au sein de chacune de ces strates pour un total de 400 .

Ce plan échantillonnal permettra de maintenir une bonne précision au niveau de l'ensemble des répondants (marge d'erreur maximale sur les proportions de 5,3\%) tout en permettant des comparaisons tant au sein du secteur secondaire que du tertiaire aussi bien qu'entre ces deux secteurs dans leur ensemble. 


\section{$5 \quad$ Résultats préliminaires de l'étude}

La cueillette des données auprès des 400 entreprises se poursuit présentement. Les seules informations dont nous disposons sont celles recueillies lors de la mise à l'essai du questionnaire auprès de 21 entreprises. Sous toute réserve, nous livrons ici quelques constatations préliminaires sur les différents paramètres étudiés. Il s'agit avant tout d'une analyse descriptive qui permet de connaître la fréquence de certaines politiques ou de certaines activités de gestion du personnel ainsi que l'incidence de certains problèmes et obstacles vécus par les PME. Cette description est suivie d'une brève analyse exploratoire qui met en relation la plupart des variables relatives à la gestion du personnel avec deux variables indépendantes à savoir la taille et le niveau de technicité des entreprises. Les entreprises ont été regroupées selon deux catégories de taille : celles entre 10 et 49 employés et celles entre 50 et 199 employés. Un deuxième regroupement a consisté à distinguer les entreprises ayant un fort niveau de technicité (soit celles dont la proportion des cadres, des professionnels et des techniciens dépassent $30 \%$ ) de celles ayant un faible niveau de technicité.

Le choix de ces variables indépendantes au niveau de l'analyse repose sur la théorie que les pratiques de gestion du personnel deviennent plus structurées avec l'augmentation du nombre des employés; elle s'appuie aussi sur l'hypothèse que la gestion des ressources humaines tend à varier selon les caractéristiques du personnel dans les entreprises. Il convient aussi de préciser que dans notre échantillon, les entreprises à forte technicité sont en majorité des petites entreprises, alors que les entreprises à faible technicité sont réparties également selon la taille.

\subsection{Caractéristiques des entreprises, du dirigeant et du personnel}

Précisons d'abord qu'il s'agit d'un échantillon aléatoire non proportionnel couvrant une gamme d'entreprises en termes de taille, de secteurs d'activité économique, et de régions du Québec.

Les 21 entreprises proviennent de toutes les régions du Québec et se répartissent assez également en nombre selon les régions urbaines (11) et les régions semi-urbaines (10). L'échantillon comprend 12 petites entreprises dont quatre de 10 à 19 employés et huit de 20 à 49 employés; il comprend aussi neuf moyennes entreprises dont cinq de 50-99 employés et quatre de $100 \mathrm{employés} \mathrm{et} \mathrm{plus.} \mathrm{Les} \mathrm{petites}$ entreprises de moins de 50 employés sont réparties également entre le secteur secondaire et tertiaire alors que les moyennes entreprises proviennent surtout du secondaire (six sur neuf).

En moyenne, ces entreprises existent depuis 24,8 années dont quatre depuis moins de 10 années, cinq depuis 11 à 20 années, cinq depuis 21 à 30 années et cinq depuis 31 années et plus. Du point de vue de l'évolution du nombre des emplois, 10 entreprises déclarent une stabilité des effectifs, sept mentionnent une augmentation alors que deux indiquent une diminution depuis les trois dernières années. Entre 1988 et 1989, il s'est créé 50 emplois nets dans ces entreprises. 
Alors que 14 entreprises déclarent une augmentation du chiffre d'affaires depuis trois ans, six autres entreprises mentionnent une stabilité. Les profits de ces entreprises se sont comportés comme suit : stabilité pour neuf d'entre elles, augmentation pour quatre autres et diminution pour quatre entreprises également. Il s'agit, pour la plupart, d'entreprises (12 sur 21) qui n'exportent pas à l'extérieur du Québec. Parmi les entreprises exportatrices, la proportion moyenne des ventes réalisées ailleurs qu'au Canada est de $33 \%$; cette proportion est de $25 \%$ dans le cas des trois entreprises exportatrices à l'extérieur du Canada.

Des 21 entreprises, neuf donnent des contrats de sous-traitance à d'autres entreprises, ce qui représente environ $10 \%$, en moyenne, des achats des entreprises. Par ailleurs, sept entreprises réalisent des contrats de sous-traitance pour le compte d'autres entreprises ce qui correspond en moyenne à plus de $68 \%$ de leur chiffre d'affaires.

Dans l'échantillon des 21 entreprises, on retrouve presqu'autant d'entreprises peu informatisées - ayant moins de cinq applications informatiques - que d'entreprises assez ou très informatisées, c'est-à-dire ayant cinq applications et plus.

La majorité des entreprises (14 sur 21) n'avaient pas de conseil d'administration ou de comité de gestion formel se réunissant sur une base régulière. Enfin, 15 des 21 entreprises étaient non syndiquées.

L'âge moyen des dirigeants de cet échantillon d'entreprises est de 45 ans. Il est composé de 6 femmes et de 15 hommes dont le diplôme obtenu est de niveau universitaire pour $10 \mathrm{~d}$ 'entre eux, de niveau collégial pour cinq autres et de niveau secondaire et primaire pour six dirigeants. Ces personnes occupent leur fonction de dirigeant dans l'entreprise depuis 11,5 années en moyenne et sont à l'emploi de cette entreprise depuis 14,1 ans.

Au total, 844 personnes, dont $84,6 \%$ à temps plein et $15,4 \%$ à temps partiel sont à l'emploi des 21 entreprises. Parmi les employés, 59,4\% sont des hommes et $40,6 \%$ sont des femmes. La composition du personnel selon l'âge est la suivante : $31,1 \%$ de moins 30 ans, $50,2 \%$ de 30 à 44 ans et $18,7 \%$ de 45 ans et plus. L'ancienneté moyenne est de 5,4 années pour l'ensemble des employés. Elle varie selon les catégories professionnelles : plus élevée pour les cadres, les professionnels et techniciens, et les employés de services $(6,5 ; 8,5 ; 8,1$ années respectivement), elle est moins élevée pour les employés de bureau, de ventes et de production $(4,6 ; 4,4$; 4,4 respectivement).

Le taux de roulement du personnel dans les entreprises observées est très important; l'on constate un taux d'accès de plus de $51 \%$ et un taux de sortie de 48 \% entre 1988 et 1989.

\subsection{Gestion en général et gestion du personnel}

La répartition du temps de travail des dirigeants parmi les fonctions suivantes de gestion est la suivante : $23 \%$ à la vente et le marketing, $22 \%$ à l'admi- 
nistration générale; $21 \%$ à la comptabilité et la finance, $18 \%$ à la production, et $15 \%$ à la gestion du personnel. Au total 10 dirigeants sur 21 n'avaient jamais participé à des activités de formation ou d'information en gestion du personnel; cinq dirigeants avaient suivi de un à trois types d'activités alors que six dirigeants avaient suivi au moins quatre types d'activités.

Selon les dirigeants interrogés, les raisons qui amènent principalement les clients à faire affaire avec leurs entreprises sont : la qualité des produits ou services $(85,7 \%$, soit 18 sur 21 , disent que c'est surtout pour cette raison), le service personnalisé de l'entreprise $(76,2 \%)$, la rapidité des délais $(60,0 \%)$, les prix plus avantageux $(47,6 \%)$; le fait d'être un des rares fournisseurs $(28,6 \%)$ passe en dernier lieu. Pour améliorer la performance de l'entreprise, la direction de ces entreprises considère que les actions suivantes en gestion du personnel ont beaucoup d'importance : améliorer l'efficacité des équipes de travail $(95,2 \%$, soit 20 sur 21 , trouvent cette action importante), informer les employés sur les objectifs de la direction et la situation de l'entreprise $(85,7 \%)$, connaître les attentes des employés $(71,4 \%)$, améliorer la polyvalence des employés $(66,7 \%)$ et faire participer les employés aux changements $(61,9 \%)$. Des actions visant à contrôler l'augmentation des salaires $(57,1 \%$, soit 12 sur 21$)$, à améliorer la mobilité interne $(55,0 \%)$, à lier le salaire au rendement ou aux profits $(52,4 \%)$, à réduire les effectifs $(23,8 \%)$, sont jugées moins importantes.

Les moyens les plus souvent utilisés par la direction des entreprises pour assurer des prix avantageux sont, en ordre d'importance, l'amélioration des compétences techniques de la main-d'œuvre $(80,0 \%$, soit 16 sur 20$)$, l'augmentation de la quantité de production ou de services $(70,0 \%)$, l'achat d'équipements plus performants $(55,0 \%)$, le maintien des coûts de main-d'œuvre le plus bas possible $(55,0 \%)$ et la recherche de matières premières ou de produits à bon compte (36,8\%).

De tous les domaines de gestion de l'entreprise, c'est dans la gestion du personnel que les dirigeants d'entreprises $(66,6 \%$, soit 12 sur 18$)$ rencontrent le plus de problèmes actuellement. Les principales difficultés mentionnées sont liées au recrutement de la main-d'œuvre ( 8 mentions), aux conditions de travail (4 mentions), au roulement et au manque de motivation du personnel (4 mentions) ainsi qu'à l'adaptation et à la formation de la main-d'œuvre (3 mentions).

L'analyse de ces résultats selon la taille et le niveau de technicité suggère que les moyennes entreprises de 50 employés et plus sont davantage préoccupées par certains aspects de la gestion du personnel, tels l'amélioration de la polyvalence, la participation des employés aux changements, l'amélioration de la mobilité interne et l'amélioration des compétences techniques. Les entreprises de faible technicité perçoivent davantage l'importance d'améliorer la mobilité interne que les entreprises à forte technicité qui, rappelons-le, sont des plus petites entreprises. Enfin, il est intéressant de constater, par ailleurs, que ce sont davantage les plus petites entreprises qui se disent aux prises avec des problèmes importants de main-d'œuvre. 


\subsection{Organisation de la gestion du personnel}

Dans l'ensemble des politiques en gestion du personnel, les seuls domaines qui font l'objet de politiques écrites sont le temps de travail et les horaires (71,4\% des entreprises, soit 15 sur 21), le mode de rémunération et d'avantages sociaux $(71,4 \%)$ ainsi que l'évaluation du personnel et la discipline $(52,4 \%)$. Les autres politiques de gestion du personnel en matière d'amélioration des conditions de travail, d'information et de consultation des employés, d'accueil et de formation, de recrutement, de relations de travail sont, pour la plupart, non-écrites. Environ le quart des entreprises (6 sur 21) n'ont aucune politique officielle en matière de planification des effectifs, de départs et de retraite, d'amélioration des conditions de travail, et de la santé et sécurité au travail. De plus, les entreprises recourent très peu à des services extérieurs en gestion du personnel sauf pour des services juridiques, la gestion de la paye et la formation du personnel, ce dernier service étant utilisé par environ $30 \%$ des entreprises.

La responsabilité de la gestion du personnel relève de plusieurs acteurs dans l'entreprise. Ainsi la moitié (soit 10 sur 21) des entreprises n'avait aucun responsable de personnel. Dans l'autre moitié des entreprises dont la gestion du personnel est confiée à une ou plusieurs personnes responsables du personnel, le pourcentage moyen du temps consacré par celles-ci à cette fonction est d'environ $50 \%$ de leur temps de travail. Dans presque tous les cas, les directeurs, les contremaîtres et les chefs assument une partie de cette fonction, ce qui occupe en moyenne $36 \%$ de leur temps de travail. De plus, les employés de secrétariat et certains professionnels (comptables et autres) consacrent respectivement une moyenne de $23,8 \%$ et $27,8 \%$ de leur temps de travail à cette fonction.

Parmi les outils de contrôle des dépenses reliés au personnel, ce sont le calcul du coût de main-d'œuvre par rapport aux autres coûts de production $(71,4 \%$ des entreprises, soit 15 sur 21, l'utilisent), le calcul des frais de main-d'œuvre par produit $(61,9 \%)$, le calcul des frais de personnel par projet ou par service $(57,1 \%)$ et certains indicateurs de productivité $(47,6 \%)$ qui sont le plus utilisés. Le calcul et le suivi du coût de la rotation du personnel, d'un changement de poste, d'un recrutement, d'un congédiement, des accidents de travail, de l'absentéisme et de la formation sont, pour la majorité des entreprises, non utilisés.

Dans $80 \%$ (soit 16 sur 21) des entreprises, la gestion du personnel ne fait pas l'objet d'un poste budgétaire spécifique. Par ailleurs, 47,6 \% (soit 10 sur 21) des entreprises ont un organigramme écrit, $42,9 \%$ ont une description de tâches écrite pour les employés et $38,1 \%$ préparent un manuel de l'employé.

Lorsque l'on tient compte de la taille des entreprises, quelques constatations ressortent de l'ensemble des résultats. Les moyennes entreprises ont plus souvent que les petites un responsable de personnel à plein temps. Elles ont recours plus souvent que les petites à des services extérieurs de formation. Parmi les outils de contrôle des dépenses de personnel, le calcul du nombre d'heures de formation et celui du coût 
unitaire d'un recrutement sont plus souvent utilisés dans la moyenne que dans la petite entreprise. Par ailleurs, on remarque que le calcul du coût de main-d'œuvre par rapport aux autres coûts de production est largement utilisé dans les entreprises de faible technicité, quelle que soit la taille et cela contrairement aux entreprises à forte technicité qui utilisent très peu ce genre d'outils.

\subsection{Planification}

Dans les petites et moyennes entreprises que nous avons observées, il se fait de la planification à court et à long terme mais surtout sous une forme non écrite et intuitive. Les plans ou études qui ont été le plus souvent faits ou fait faire par les entreprises sont : la planification budgétaire ( $76,2 \%$ soit 16 sur 21$)$, l'étude de marché $(57,2 \%)$, l'étude de rentabilité $(57,2 \%)$ et la planification stratégique $(47,6 \%)$. Par ailleurs, la planification budgétaire et l'étude de marché sont plus souvent réalisées dans la moyenne que dans la petite entreprise. C'est dans le domaine de l'implantation des changements technologiques, de l'estimation des besoins de main-d'œuvre et des besoins de formation que l'exercice de planification est le moins souvent pratiqué (environ le tiers, seulement, des entreprises le font).

\subsection{Recrutement}

Les principales sources de recrutement utilisées par les entreprises sont dans l'ordre : les journaux locaux et nationaux (61,9\%, soit 13 sur 21$)$, les candidatures spontanées, les candidats recommandés par les employés ainsi que les écoles, collèges et universités (47,6\% des entreprises les utilisent).

Les techniques de sélection de personnel les plus souvent utilisées sont l'entrevue en personne, l'analyse des formulaires de demande d'emploi et des curriculum vitae ainsi que la probation. $L$ 'analyse des demandes d'emploi et les tests sont plus souvent pratiqués dans la moyenne que la petite entreprise. Il en est de même pour les entreprises à forte technicité. Dans la grande majorité des entreprises $(81 \%$, soit 17 sur 21), des références sont demandées aux candidats potentiels; celles-ci sont toujours vérifiées dans seulement $23,8 \%$ des cas, la plupart l'étant occasionnellement $(47,6 \%)$ ou pas du tout $(9,5 \%)$.

Plus de 71,4\% (15 sur 21 ) des entreprises signalent des difficultés à recruter la main-d'œuvre dont elle a besoin. Cette difficulté est le plus souvent imputée à une déficience de formation des candidats.

\subsection{Formation}

La formation est vue par la plupart des dirigeants comme un moyen de conserver et d'accroître les compétences techniques de l'entreprise, comme un instrument utile pour améliorer la productivité de l'entreprise et même comme un élément de satisfaction et de motivation des employés. Au total 175 employés, soit 
20,7 de l'ensemble des employés des entreprises ont reçu de la formation en cours de travail alors que 141 employés, soit 16,7\% de l'ensemble, se sont vus offrir de la formation organisée. Cependant, en termes de jours de formation reçue, les activités de formation en cours de travail demeurent prépondérantes. Ainsi, dans l'ensemble, il s'est donné en moyenne, par année, par employé 20,2 jours de formation en cours de travail et 8,9 jours de formation organisée.

De façon générale, les entreprises comptent sur leurs propres ressources pour satisfaire les besoins de formation de la main-d'œuvre, les autres ressources le plus souvent utilisées étant les établissements publics d'enseignement, les fournisseurs d'équipements et les consultants privés.

La plupart des entreprises (65\%, soit 13 sur 20) éprouvent des difficultés à réaliser des activités de formation organisée. Ces difficultés sont liées au remplacement des personnes à former, aux fluctuations dans la production qui permettent difficilement de planifier ce genre d'activités et, finalement, aux coûts de formation trop élevés pour l'entreprise.

Les dirigeants des entreprises indiquent que certaines activités de formation pourraient être utiles dans la gestion du personnel en matière, notamment, de direction du personnel (relations humaines, communication et motivation) et de gestion des relations et des conditions de travail.

D'autres constatations ressortent en contrôlant la taille des entreprises. Les moyennes entreprises manifestent une plus grande préoccupation que la petite pour les activités de formation. La direction des moyennes entreprises considère, davantage que les petites, l'importance de la formation comme moyen de conserver et d'accroître les compétences techniques de l'entreprise et comme moyen d'intégration à la vie et aux valeurs de l'entreprise. On constate, d'ailleurs, que la petite entreprise offre moins d'activité de formation organisée que l'entreprise de taille moyenne. C'est toutefois dans l'entreprise moyenne que l'on rencontre le plus de difficultés à réaliser des activités de formation organisée liées aux problèmes de remplacement du personnel à former.

\subsection{Rémunération}

Les moyens les plus souvent utilisés pour déterminer le salaire sont l'ajustementà la capacité de payer de l'entreprise, l'établissement d'une structure salariale, l'ajustement aux pratiques des entreprises concurrentes et la négociation de conventions collectives. Très peu d'entreprises disent se référer aux annonces placées dans les journaux, aux résultats d'enquêtes sur la rémunération, au taux du salaire minimum et à l'évolution du salaire moyen au Québec, ou encore procèdent à des ententes individuelles avec chaque employé.

Les entreprisesà forte technicité utilisent plus souvent l'évaluation formelle des emplois et une structure salariale pour déterminer les salaires que les entreprises 
de niveau de technicité plus faible. Ces dernières se fient beaucoup plus aux intuitions et connaissances de la direction. De plus, la moyenne entreprise se réfere plus que la petite au taux du salaire minimum et à l'évolution du salaire moyen pour établir la rémunération.

\subsection{Information et participation}

La direction des entreprises fournit en général à ses cadres des informations sur la mission de l'entreprise, sa clientèle, et les changements prévus; elle leur signifie également les attentes de la direction, les politiques du personnel et les informe sur l'évaluation du rendement ainsi que les décisions du conseil d'administration. Dans le cas des autres employés, l'évaluation du rendement, la santé et sécurité dans l'entreprise, les attentes de la direction, et les politiques du personnel sont les principaux objets d'information.

Les moyens de communication privilégiés par les entreprises sont des réunions générales, des rencontres sociales, des réunions ponctuelles pour la résolution de problèmes et le recueil des opinions des employés.

\section{Conclusion}

Ce portrait, quoique fragmentaire, des pratiques de gestion du personnel des PME au Québec nous permet de préciser certaines hypothèses soulevées par les théoriciens et les praticiens dans ce domaine. Bien que ce tableau général ne puisse être considéré comme représentatif de la situation des PME au Québec, l'échantillon étant très petit et non proportionnel, il n'en demeure pas moins que la grande variété des entreprises observées fait ressortir des tendances qui seront intéressantes à vérifier auprès d'un échantillon plus large de 400 petites et moyennes entreprises.

La place de la gestion des ressources humaines dans la gestion des PME diffère selon la taille et le degré de technicité des entreprises. Alors que la préoccupation majeure de l'ensemble des PME observées, est celle de la qualité et du service personnalisé de l'entreprise, les dirigeants de moyenne entreprise, plus que la petite, sont davantage enclins à structurer minimalement la gestion du personnel, à utiliser des outils de contrôle des dépenses du personnel, à faire de la planification budgétaire, à formaliser leurs méthodes de recrutement et de rémunération et à développer des activités de formation organisée. Par ailleurs, les données suggèrent aussi que le degré de technicité des entreprises a une influence sur les politiques et pratiques de gestion du personnel. Les entreprises à forte technicité ont plus tendance à formaliser leurs techniques de recrutement et l'établissement de la rémunération.

Par ailleurs, c'est dans le domaine de la gestion du personnel que les PME rencontrent le plus de difficultés. Les problèmes de recrutement, de rétention et de roulement de la main-d'œuvre, liés à des conditions de travail souvent précaires, sont 
des sujets importants de préoccupation des dirigeants. On remarque que ces entreprises sont peu outillées pour planifier les besoins de main-d'œuvre, de formation et de changements technologiques, l'activité de planification budgétaire et d'étude de marché étant prépondérante. Aussi, le contrôle des dépenses de main-d'œuvre est limité à des indicateurs de productivité, et $n$ 'inclut que très rarement des outils liés aux coûts de rotation, de recrutement, d'absentéisme et de formation du personnel. De même on remarque une absence plus fréquente de politiques officielles en matière de planification des besoins de main-d'œuvre, de départs et de retraites.

Dans cette analyse préliminaire, nous avons cherché à contrôler l'influence de la taille et de la technicité des entreprises. Plusieurs autres facteurs, tels l'âge de l'entreprise, l'âge de la gestion, l'informatisation, la stratégie d'entreprise aussi bien que le profil des dirigeants, leurs valeurs et leur vision peuvent influencer la gestion du personnel dans les PME. C'est ce type d'analyse que nous entendons poursuivre.

L'importance des PME dans l'économie québécoise justifie grandement qu'une étude sur la gestion des ressources humaines soit entreprise. En effet, la PME au Québec contribue de façon déterminante à la création d'emploi, compensant ainsi le recul observé dans les grandes entreprises. Plus souple, plus flexible, elle réussirait, à certains égards, à mieux s'adapter aux changements économiques et sociaux que la grande entreprise.

Les PME connaissent aussi, toutefois, un taux élevé de décès, ce qui entraîne des pertes d'emploi. De plus, les emplois offerts dans les PME sont souvent précaires et moins rémunérés que ceux de la grande entreprise. On sait par ailleurs que la PME connaît des problèmes importants de gestion du personnel. La forte présence des jeunes dans les PME et l'émergence d'une nouvelle génération d'entrepreneurs plus instruits et plus ouverts sont des phénomènes qu'il est pertinent d'approfondir. Il apparaît donc utile d'engager une réflexion sur la gestion des ressources humaines et la place qu'elle occupe dans la gestion des petites et moyennes entreprises, sur les types de stratégie de GRH en relation avec les stratégies d'entreprise, les profils de dirigeants et de personnel.

\section{BIBLIOGRAPHIE}

Audet, M., Bélanger, L. et Jacques, F., (1987), «Place, importance et pratiques de la gestion des ressources humaines dans les entreprises québécoises», Québec, Département des relations industrielles, Université Laval.

Beer, M., Spector, B., Lawrence, P.R., Mills, D.G. et Walton, R.E., (1984), Managing Human Assets, New York, The Free Press.

Bélanger, L., Benabou, C., Foucher, R. et Petit, A., (1988), Gestion stratégique des ressources humaines, Montréal, Gaétan Morin éditeur.

Benoit, C. et Rousseau, M.-D., (1989), «Gestion des ressources humaines dans les PME au Québec», Rapport synthèse d'entrevues auprès des personnes-ressources», Montréal, Ministère de la Main-d'œuvre et de la Sécurité du revenu. 
Besseyre des Horts, C.-H., (1987), «Typologies des pratiques de gestion des ressources humaines», Revue française de gestion, novembre-décembre, p. 149-155.

Besseyre des Horts, C.-H., (1988), Vers une gestion stratégique des ressources humaines, Paris, Les Éditions d'Organisation.

D’Amboise, G. et Gasse, Y., (1982), «Les défis administratifs quotidiens dans les PME québécoises», Commerce, mai.

D'Amboise, G. et Muldowney, M., (1984), «Specific theory for small firms : Attemps and requirements», 1984 Rencontre de Saint-Gall of the Swiss Research for Small Business, Zermatt, Suisse.

Fombrun, C., Tichy, N.M. et Devanne, M.A., (1984), Strategic Human Resource Management ${ }_{2}$ New York, Wiley.

Guérin, G. et Bouteiller ,D., (1988), «La philosophie de gestion des ressources humaines : un outil de gestion ?», Département de relations industrielles, Université de Montréal, document 88-08.

Laroche, G., (1988), «La PME au Québec : une manifestation de dynamisme économique du Québec», Gouvernement du Québec, ministère de la Main-d'œuvre et de la Sécurité du revenu, p. 11-36.

Lauzier, P.J. et Thompson, P., (1987), «Poste à combler dans le secteur de l'entreprise indépendante (deuxième partie) - Analyse provinciale : le Québec», Montréal, Fédération canadienne de l'entreprise indépendante.

Mahé de Boislandelle, H., (1988), Gestion des Ressources Humaines dans les PME, Paris, Ed. Economica.

Ministère de l'Industrie, du Commerce et de la Technologie, (1988), Les PME au Québec. État de la situation, Québec, Direction des communications.

Thompson, P., (1989), «Des compétences pour l'avenir : la petite entreprise et la formation professionnelle au Canada», Toronto, F.C.E.I.

Tremblay, D.G., Les réalités et les enjeux de la formation professionnelle, Montréal (à paraître).

Werther, W.B. jr., Davis, K. et Lee-Gosselin, H., (1985), La gestion des ressources humaines, Montréal, McGraw-Hill Éditeurs.

Wils, T., Labelle, C., Guérin, G. et Le Louarn, J.-Y., (1989), «La gestion stratégique des ressources humaines. Un reniement du rôle social de l'entreprise ?», Relations industrielles, vol. 44, no 2, p. 354-375. 